\title{
Real-World Treatment Patterns for Hormone Receptor- Positive, Human Epidermal Growth Factor Receptor 2-Negative Advanced Breast Cancer in Europe and the United States
}

Rita Caldeira · Mark Scazafave

Received: September 5, 2016 / Published online: October 27, 2016

(C) The Author(s) 2016. This article is published with open access at Springerlink.com

\begin{abstract}
Introduction: Clinical guidelines generally recommend endocrine therapy over chemotherapy for hormone receptor-positive advanced breast cancer (unless life-threatening metastases are present). This study aimed to assess the real-world treatment patterns of patients with hormone receptor-positive advanced breast cancer in Europe and the United States.
\end{abstract}

Methods: Treatment patterns in Europe (France, Germany, Italy, Spain, and the UK) and the United States from January 2012 to December 2014 were investigated using a patient record database (Global Oncology Monitor $\odot)$. Sample data were projected to the wider clinical population to provide running annual estimates every 3 months.

Enhanced content To view enhanced content for this article go to http://www.medengine.com/Redeem/ B217F060485A77D5.

R. Caldeira $(\square)$

Ipsos Healthcare, London, UK

e-mail: Rita.Caldeira@ipsos.com

M. Scazafave

Ipsos Healthcare, Mahwah, NJ, USA
Results: Sample sizes ranged from 1272 to 1640 patients in Europe and from 2225 to 2760 patients in the United States. Across all lines of therapy, $37-43 \%$ (Europe) and $45-50 \%$ (United States) of patients received chemotherapy. More patients received endocrine therapy than chemotherapy as first-line treatment for advanced breast cancer (Europe: $51-54 \%$ vs. 33-35\%; United States: $53-60 \%$ vs. $34-42 \%)$. In contrast, endocrine therapy-only regimens were given less commonly than chemotherapy-only regimens in the third-line setting in both Europe and the United States.

Conclusions: Chemotherapy is used extensively in routine clinical practice for hormone receptor-positive, human epidermal growth factor receptor 2-negative advanced breast cancer. The results also suggest that the treatment patternsin Europe and the United States are qualitatively different.

Funding: Ipsos Healthcare and AstraZeneca.

Keywords: Chemotherapy; Endocrine therapy; Guidelines; Hormone receptor-positive breast cancer; Quality of care; Treatment patterns 


\section{INTRODUCTION}

Clinical guidelines produced by the joint European School of Oncology/European Society of Medical Oncology task force and by the National Comprehensive Cancer Network recommend that patients with hormone receptor (HR)-positive, human epidermal growth factor receptor (HER)2-negative advanced breast cancer should be treated with endocrine therapy, including when asymptomatic visceral metastases are present $[1,2]$. Cytotoxic chemotherapy has proven efficacy but, due to its tolerability profile, should be reserved for patients with rapidly progressing disease and/or endocrine resistance $[1,2]$. Recommended endocrine therapies include the selective estrogen receptor modulator tamoxifen; third-generation aromatase inhibitors (AIs) anastrozole, letrozole, and exemestane; and the selective estrogen receptor downregulator fulvestrant [3].

Limited data are available for assessing the use of these treatments in routine practice. We report real-world use of these therapies for the treatment of postmenopausal patients with HR-positive, HER2-negative advanced breast cancer in Europe and the United States.

\section{METHODS}

\section{Study Design and Participants}

The Global Oncology Monitor $\odot$ (Ipsos Healthcare, London, UK)—a large physician-based syndicated patient record database-was used to identify treatments given to postmenopausal patients with HR-positive, HER2-negative locally advanced/ metastatic breast cancer (stage IIIb/stage IV) in five European countries (France, Germany, Italy, Spain, and the UK) and the United
States. Annually, approximately 1300 oncology physicians (representing over 105,000 patients) continuously complete patient case histories for approximately 5-30 patients currently receiving treatment and seen by the physician in the previous month. The majority of records were collected online and the remainder via paper forms. Responding physicians were the primary decision-maker for included patients. The Global Oncology Monitor $\odot$ is validated with market sizing studies every 2 years to ensure that the size and representativeness of the physician sample reflects the wider population of relevant treating physicians. This article is based on previously conducted studies, and does not involve any new studies of human or animal subjects performed by any of the authors.

Patient records were reviewed for type and duration of treatment. The evaluation period ran from January 1, 2012, until December 31, 2014. Sample data based on annual market sizing were projected to the wider clinical population to provide running annual estimates every 3 months. Projections were adjusted for physician, practice type, line of therapy, and cancer type and stage to reflect clinical practice.

Projected treatments were allocated to the following mutually exclusive categories: chemotherapy only; "biologics" only (including palbociclib, everolimus, and trastuzumab); endocrine therapy only; chemotherapy plus biologics; chemotherapy plus endocrine therapy; and biologics plus endocrine therapy. Subgroup analyses were performed on the following groups of patients receiving first-line treatment for locally advanced/metastatic breast cancer: patients receiving their first ever drug treatment for breast cancer within the locally advanced/ metastatic setting, who had been diagnosed 


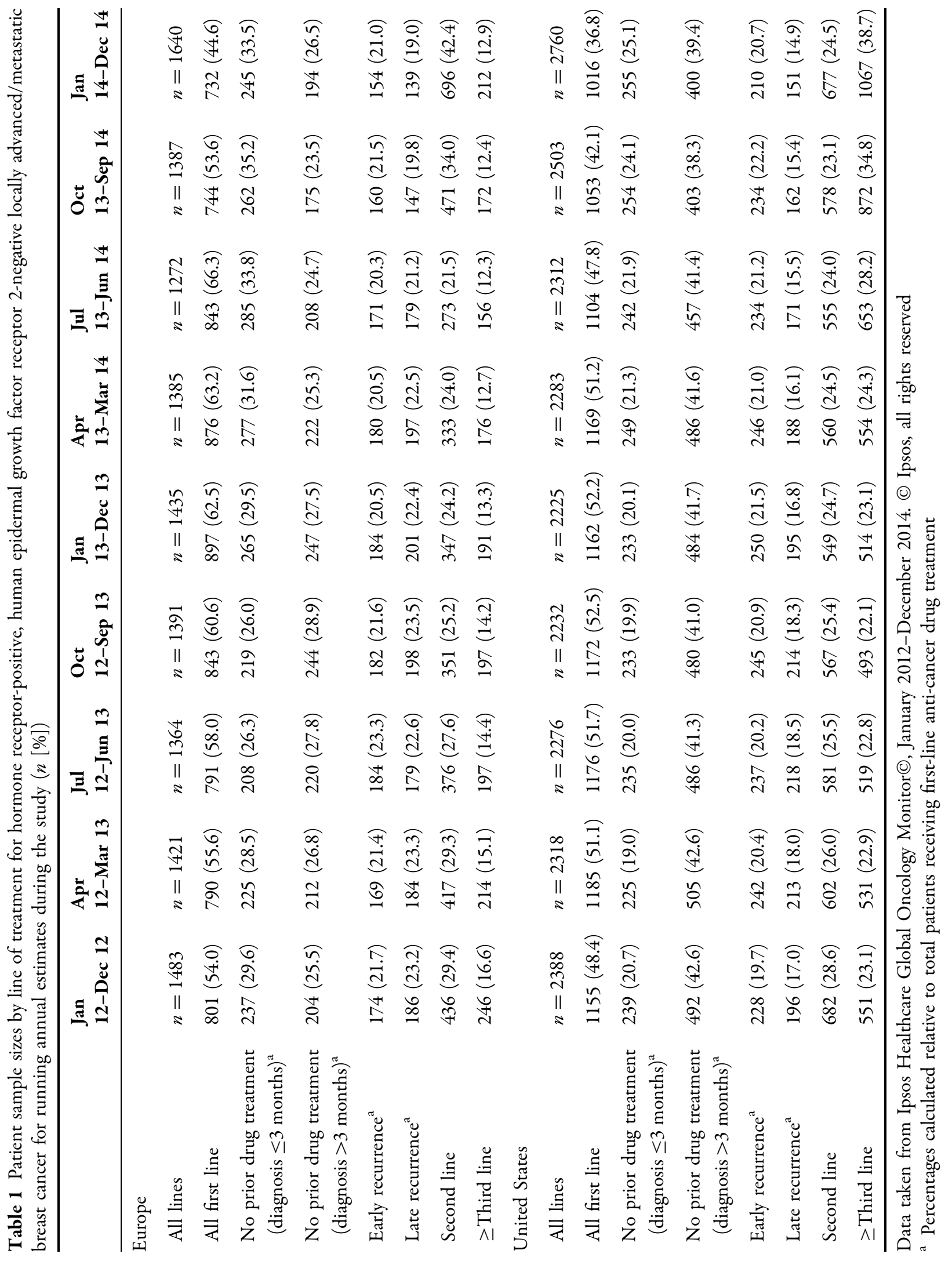


within the previous 3 months ("no prior drug treatment [diagnosis $\leq 3$ months]"); patients receiving their first ever drug treatment for breast cancer but who had been diagnosed more than 3 months previously ("no prior drug treatment [diagnosis $>3$ months]"); patients who had previously received drug treatment for early breast cancer and experienced disease progression within $\leq 12$ months of treatment ("early recurrence"); and patients who had received drug treatment for early breast cancer and experienced disease progression $>12$ months after treatment ("late recurrence"; this group also included patients whose time to recurrence was unknown). Results for this observational report are descriptive, with no additional statistical analysis. Values are reported as ranges of running annual estimates unless otherwise stated.

\section{RESULTS}

The representative sample of physicians in the United States comprised those in office or private clinics (68\%), universities or teaching hospitals (17\%), general hospitals (11\%) and comprehensive cancer centers (5\%). In Europe, physicians were at university or teaching hospitals (45\%), general hospitals (29\%), comprehensive cancer centers (18\%) or office/ private clinics (7\%). The sample sizes used every 3 months ranged from 1272 to 1640 patients in Europe (France, Germany, Italy, Spain, and the UK) and from 2225 to 2760 patients in the United States (Table 1). All patients were being treated with an anti-cancer drug at the time their data was recorded. Among these, 45-66\% (Europe) and 37-53\% (United States) of patients were receiving their first line of anti-cancer drug treatment for advanced disease during the evaluation period; $22-42 \%$ (Europe) and
Fig. 1 Projected treatment regimen use among patients with hormone receptor-positive, human epidermal growth factor receptor 2-negative locally advanced/metastatic breast cancer in Europe and the United States by line of therapy for advanced disease. Data taken from Ipsos Healthcare Global Oncology Monitor(c), January 2012-December 2014 (see Table 1 for sample sizes). (C) Ipsos, all rights reserved

23-29\% (United States) were receiving their second-line treatment; and $12-17 \%$ (Europe) and $22-39 \%$ (United States) were receiving third- or later-line treatment.

When assessed across all lines of therapy, running annual projections during the evaluation period indicated that endocrine therapy-only and chemotherapy-only regimens were the most commonly prescribed treatments, with each category accounting for approximately $40 \%$ of projected treatments in Europe (43-47\% and 37-43\%, respectively) and the United States $(39-42 \%$ and $45-50 \%$, respectively; Fig. 1). Chemotherapy plus biologics and biologics plus endocrine therapy were received by $6-8 \%$ and $1-9 \%$ of patients in Europe, and $0-2 \%$ and $4-11 \%$ of patients in the United States, respectively. Fewer than $4 \%$ of patients received biologics only or chemotherapy plus endocrine therapy in either Europe or the United States.

In the first-line setting, endocrine therapy-only regimens were given more commonly than chemotherapy-only regimens in Europe (51-54\% vs. 33-35\%) and the United States $(53-60 \%$ vs. $34-42 \%)$ during the evaluation period (Fig. 1). In the third-line setting, endocrine therapy-only regimens were given less commonly than chemotherapy-only regimens in both Europe and the United States. There were no clear differences in patterns of use between endocrine-only and chemotherapy-only regimens in the second-line setting. 

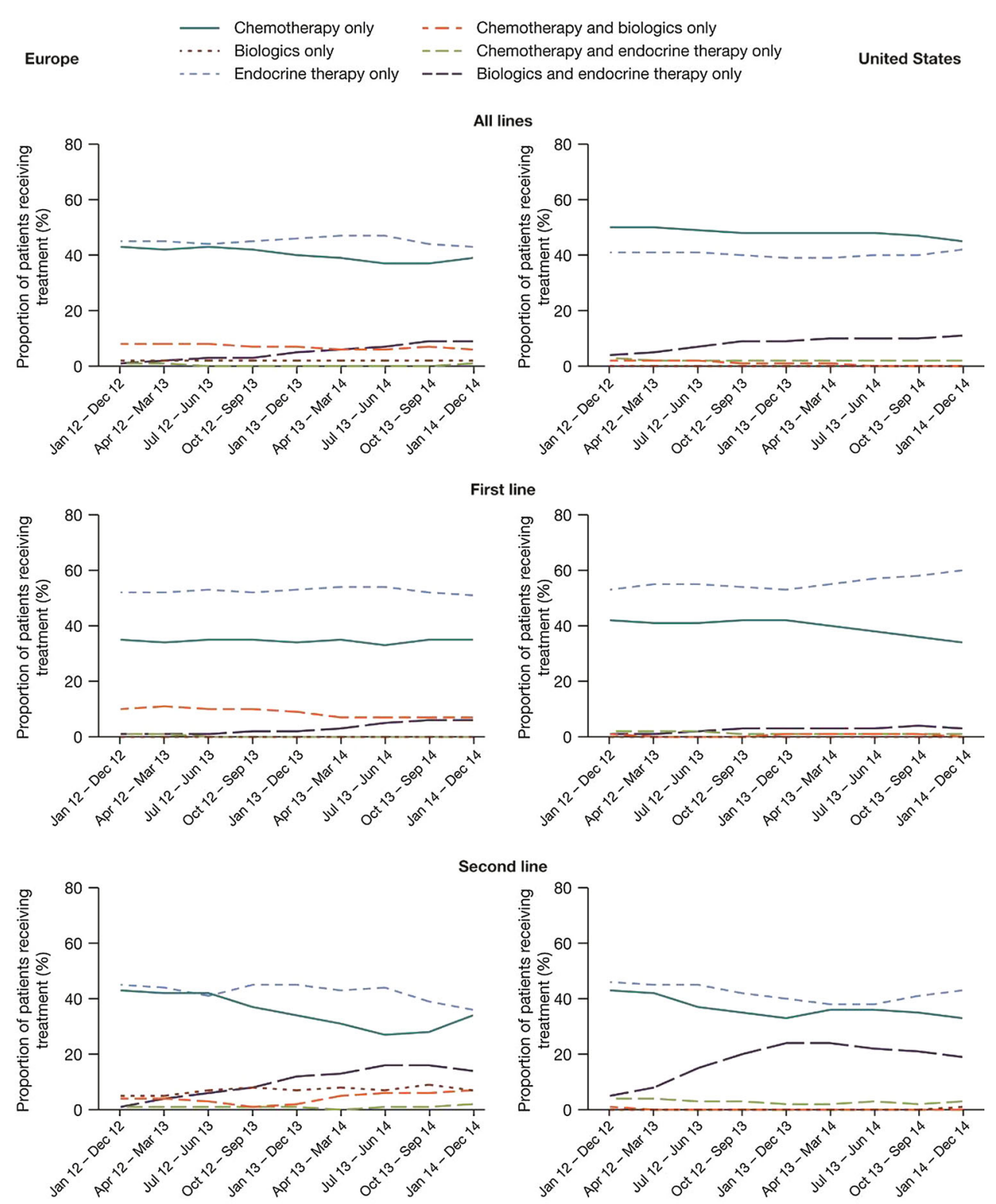

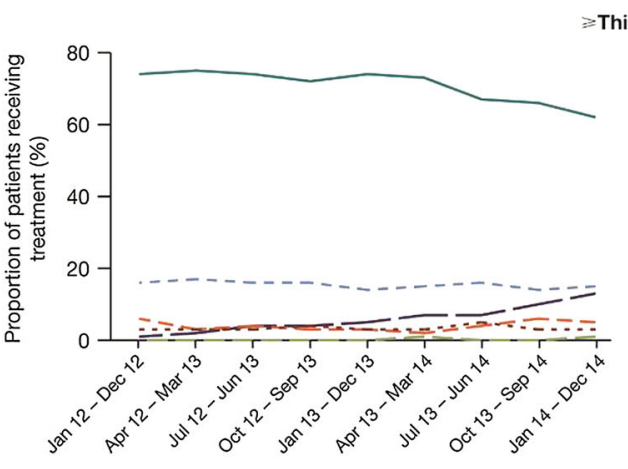

3-monthly running annual estimate

$\geqslant$ Third line

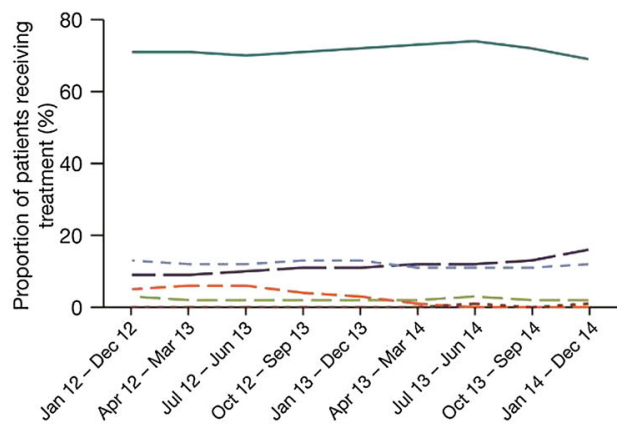

3-monthly running annual estimate 

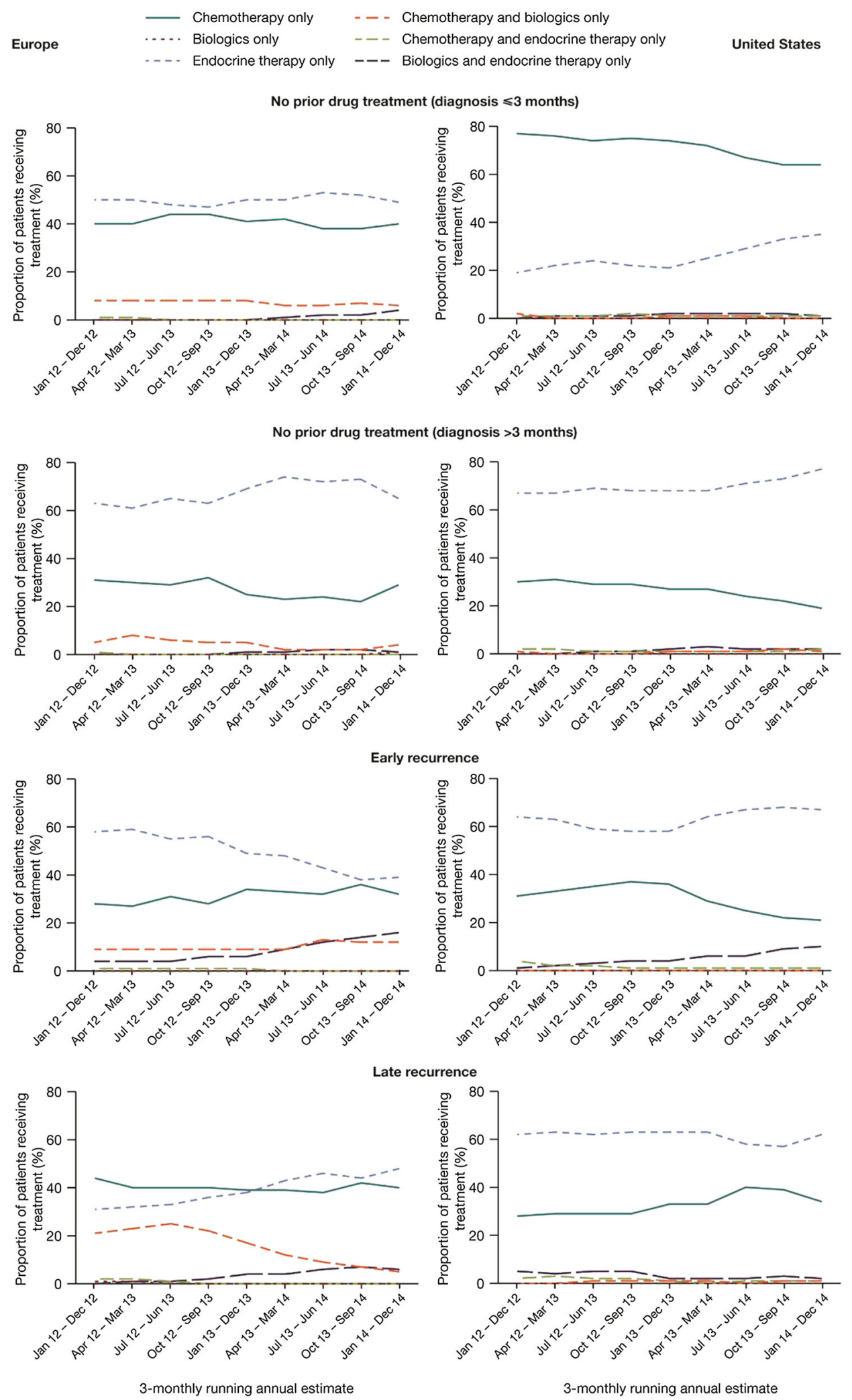
4Fig. 2 Projected treatment regimen use among patients receiving first-line treatment for hormone receptor-positive, human epidermal growth factor receptor 2-negative locally advanced/metastatic breast cancer in Europe and the United States by category of advanced disease. Data taken from Ipsos Healthcare Global Oncology Monitor(C) January 2012-December 2014 (see Table 1 for sample sizes). (C) Ipsos, all rights reserved

In Europe, when assessed by category, first-line treatment patterns appeared similar between chemotherapy-only and endocrine therapy-only regimens in patients with late-recurrent disease (Fig. 2). Patients in the no-prior-drug-treatment (diagnosis $\leq 3$ months) and no-prior-drug-treatment (diagnosis $>3$ months) subgroups more commonly received endocrine therapy-only than chemotherapy-only regimens. In the early-recurrence subgroup, the proportion of patients who received endocrine therapy only decreased over the evaluation period, reaching a level similar to patients who received chemotherapy-only regimens. However, there was also an increase over this time in the proportion of patients in the early-recurrence subgroup who received endocrine therapy plus biologics.

In the United States, fewer patients with no prior drug treatment (diagnosis $\leq 3$ months) received first-line endocrine therapy-only versus chemotherapy-only regimens (Fig. 2). A higher proportion of patients received endocrine therapy-only compared with chemotherapy-only regimens in the no-prior-drug-treatment (diagnosis $>3$ months), early-recurrence, and late-recurrence subgroups.

\section{DISCUSSION}

Data from this analysis of a patient record database suggest that a substantial proportion of patients with HR-positive, HER2-negative advanced breast cancer receive chemotherapy. A previous US database analysis from 2002 to 2012 observed similar values for the proportion of patients receiving chemotherapy (40\%) and endocrine therapy (60\%) for first-line treatment of HR-positive, HER2-negative metastatic breast cancer [4]. This study extends these observations to Europe, and suggests that these patterns have remained consistent in subsequent years. Although the design and scope of this study prevent any firm conclusions from being drawn regarding treatment use versus guideline recommendations (and all treatment decisions should be made on an individualized basis), it is possible that some physicians could be better educated on the benefits of endocrine therapy compared with chemotherapy for HR-positive advanced breast cancer. Use of endocrine therapy in combination with biologics appeared to increase during the evaluation period. This may reflect the approval of everolimus in 2012 by both the European Medicines Agency [5] and the US Food and Drug Administration [6] for use in combination with exemestane in patients with HR-positive, HER2-negative advanced breast cancer that has progressed during prior treatment with AIs.

The data presented here suggest important qualitative differences in first-line treatment of HR-positive, HER2-negative advanced breast cancer between the subgroups of patients analyzed and between Europe and the United States. For example, chemotherapy regimens were used preferentially in the first-line setting for advanced breast cancer in patients in the United States with no prior drug treatment (diagnosis $\leq 3$ months). However, in Europe, a greater proportion of patients were given endocrine therapy than chemotherapy in this setting. A previously published study has also 
found European practice patterns to be generally consistent with guideline recommendations [7]. However, to our knowledge, there have been no published studies that have investigated the differences in practice patterns for advanced breast cancer between the United States and Europe. Therefore, initial study could prompt more in-depth investigation.

It is possible that the apparent low utilization of endocrine therapy for advanced breast cancer, especially as second- and third-line therapies, may be because patients develop rapidly progressing disease after first-line treatment (possibly also with the presence of life-threatening visceral metastases), making them candidates for chemotherapy. Alternatively, physicians may have reasons to expect further lines of endocrine therapy to lack benefit due to the development of endocrine resistance [8], or they may be willing to concede the increased toxicity with chemotherapy with an expectation of achieving more effective disease control [4]. This could be interpreted as a belief by some physicians that the risk-benefit profile of endocrine therapies in the second- and third-line settings are not superior to chemotherapy [4]. However, these speculations extend beyond the current study, and the relevant data regarding physicians' motivations for therapy choice were not collected.

These results should be interpreted with caution and within the limitations of this patient database analysis. No statistical analyses were incorporated into this study, which was intended to be descriptive. Furthermore, data were not available within the scope of the study to fully define the patient population that may be eligible for chemotherapy according to clinical guidelines (i.e., with rapidly progressive disease or proven endocrine resistance).

\section{CONCLUSION}

Based on the data presented, we conclude that chemotherapy appears to be used extensively for the treatment of hormone receptor-positive advanced breast cancer in routine clinical practice in Europe and the United States.

\section{ACKNOWLEDGMENTS}

This research was funded and conducted by Ipsos Healthcare. Article processing charges were funded by AstraZeneca. AstraZeneca had the opportunity to review and provide comments on drafts of the publication prior to submission. Medical writing support for this article was provided by Dr. Martin Bell of Complete Medical Communications, funded by AstraZeneca. All authors had full access to all of the data in this study and take complete responsibility for the integrity of the data and accuracy of the data analysis. All named authors meet the International Committee of Medical Journal Editors (ICMJE) criteria for authorship for this manuscript, take responsibility for the integrity of the work as a whole, and have given final approval of the version to be published.

Disclosures. R. Caldeira is an employee of Ipsos Healthcare. M. Scazafave is an employee of Ipsos Healthcare. AstraZeneca is a client of Ipsos Healthcare.

Global Oncology Monitor, including data contained within this article from the Global Oncology Monitor, used with permission. (c) Ipsos 2016, all rights reserved. 
Compliance with Ethics Guidelines. This article is based on previously conducted studies, and does not involve any new studies of human or animal subjects performed by any of the authors.

Data Availability. All data generated or analyzed during this study are included in this published article.

Open Access. This article is distributed under the terms of the Creative Commons Attribution-NonCommercial 4.0 International License (http://creativecommons.org/licenses/ by-nc/4.0/), which permits any noncommercial use, distribution, and reproduction in any medium, provided you give appropriate credit to the original author(s) and the source, provide a link to the Creative Commons license, and indicate if changes were made.

\section{REFERENCES}

1. Cardoso F, Costa A, Norton L, et al. ESO-ESMO 2nd international consensus guidelines for advanced breast cancer (ABC2). Ann Oncol. 2014;25:1871-88.

2. National Comprehensive Cancer Network. NCCN Clinical Practice Guidelines in Oncology Version
2.2015: Breast Cancer. http://www.nccn.org/ professionals/physician_gls/PDF/breast.pdf. Accessed July 23, 2016.

3. Sainsbury R. The development of endocrine therapy for women with breast cancer. Cancer Treat Rev. 2013;39:507-17.

4. Swallow E, Zhang J, Thomason D, Tan RD, Kageleiry A, Signorovitch J. Real-world patterns of endocrine therapy for metastatic hormone-receptor-positive $(\mathrm{HR}+)$ /human epidermal growth factor receptor-2-negative (HER2-) breast cancer patients in the United States: 2002-2012. Curr Med Res Opin. 2014;30:1537-45.

5. European Medicines Agency. Everolimus approval. http://www.ema.europa.eu/docs/en_GB/document_ library/Summary_of_opinion/human/001038/WC500 129092.pdf. Accessed July 23, 2016.

6. US Food and Drugs Administration. Everolimus approval. http://www.fda.gov/Drugs/InformationOn Drugs/ApprovedDrugs/ucm313008.htm. Accessed July 23, 2016.

7. Andre F, Neven P, Marinsek N, et al. Disease management patterns for postmenopausal women in Europe with hormone-receptor-positive, human epidermal growth factor receptor-2 negative advanced breast cancer. Curr Med Res Opin. 2014;30:1007-16.

8. Macalalad AR, Hao Y, Lin PL, et al. Treatment patterns and duration in post-menopausal women with $\mathrm{HR}+$ / HER2- metastatic breast cancer in the US: a retrospective chart review in community oncology practices (2004-2010). Curr Med Res Opin. 2015;31:263-73. 